\title{
Approches intégrées et planification urbaine : défis et enjeux de la participation et de l'intégration
}

\author{
Geneviève Cloutier et Gilles Sénécal ${ }^{1}$ \\ INRS-Urbanisation, Culture et Société
}

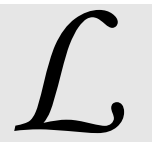

e terme d'approche intégrée fait florès. Son emploi répété dans les milieux de la planification et de l'aménagement renvoie à la volonté d'aborder de manière coordonnée un ensemble de questions jusque-là traitées de façon sectorielle. Repris dans l'élaboration de politiques et de programmes publics, il correspond aux processus par lesquels des acteurs publics, communautaires et privés tentent de structurer des réseaux et des collaborations dans le but de planifier et d'agir à l'intérieur d'un milieu donné. La mise en œuvre de ces processus nécessite des arrangements organisationnels particuliers permettant de réunir des acteurs issus de différentes organisations ou institutions, bien souvent engagées à différentes échelles spatiales, afin de saisir simultanément des thématiques particulières, conçues et gérées sur une base sectorielle ou en silo, pour reprendre une expression de plus en plus répandue pour désigner le confinement des organisations à l'intérieur de mandats pointus. En milieu urbain, le terme d'approche intégrée recouvre les initiatives dans lesquelles convergent différents réseaux d'acteurs associés à différentes fonctions urbaines et engagés à traiter de façon concertée des problèmes comme la relance des quartiers en crise ou la ségrégation socio-spatiale. De telles approches intégrées témoignent ainsi d'une grande ambition, celle de nouer des partenariats très larges, susceptibles de transcender les intérêts particuliers et de déborder les logiques organisationnelles et disciplinaires ancrées dans des pratiques anciennes afin de parvenir à des solutions concertées qui soient partagées et articulées autour d'un intérêt général socialement construit. C'est dans cette optique que les initiateurs du programme de Revitalisation urbaine intégrée (RUI) de la Ville de Montréal ont récemment prévu, dans le quartier Sainte-Marie, «d'intervenir de manière simultanée, de façon intégrée et ciblée, sur toutes les dimensions d'un quartier et de sa communauté $»^{2}$.

À l'heure où les décideurs, les planificateurs de même que tous les acteurs sociaux engagés dans des stratégies de développement (urbain, social, économique, etc.) sont invités à " s'intégrer », peut-on justement poser la question de la pertinence théorique de telles approches? Par delà le discours sur l'intégration destiné peut-être à satisfaire des intellectuels ou des gestionnaires publics qui dénoncent justement le traitement en silo des problèmes sociaux et urbains, peut-on s'interroger sur les pratiques qui découlent des approches intégrées? Le but de cet article est d'aborder la portée de l'intégration et de la participation à l'intérieur des pratiques de planification. À cet égard, un retour sur les théories de planification s'impose. Ensuite, il conviendra de situer l'approche intégrée vis-à-vis une question précise, soit celle de la revitalisation des quartiers anciens, en prenant pour exemple l'expérience de la RUI dans un quartier montréalais.

\section{Planification urbaine, participation et société pluraliste}

Le planificateur rationnel possédant le monopole de l'expertise et chargé d'assurer l'ordre urbain n'est plus. Il s'est éclipsé alors que s'affirmait une conception plus sociale et culturelle de la ville. Le planificateur s'est ainsi mué en un acteur social pressé d'agir 
dans des milieux diversifiés et complexes, dorénavant outillé pour traiter avec des forces sociales bien organisées et rompu à l'art de participer à la vie communautaire. Depuis les trente dernières années, en effet, le planificateur participe aux débats sociaux et interagit avec des personnes affirmant leur authenticité. Dans ces forums ouverts aux débats, chacun peut faire valoir des intérêts, des opinions et des représentations qui lui sont propres. C'est en cela que réside le grand enjeu actuel de la planification : le professionnel qui en est chargé doit s'ouvrir à la diversité et accepter qu'une certaine subjectivité entoure l'acte d'aménager. Car on conçoit dorénavant l'urbain comme un mode de vie ainsi qu'un espace de consommation et de production de symboles.

Dans cette partie, on discute donc des thèses développées par Beauregard, Innes et Fishman sur la planification de même que de l'interprétation culturelle des modes de vie urbains défendue par Zukin ${ }^{3}$. La ville y est comprise comme un ensemble abstrait d'éléments qui permet à ses habitants de créer des liens, de s'organiser et de mettre en place de nouvelles pratiques sociales. Dans cette optique, la représentation de la ville varie selon les contenus, les valeurs et les intentions de chacun. Dès lors, le planificateur est sommé de tenir compte de ces représentations différenciées ainsi que des besoins spécifiques lorsqu'il est question de prévoir le devenir de la ville et de ses quartiers. À un point tel qu'ils sont dorénavant nombreux, les planificateurs, à reconnaître l'intérêt d'associer, de près ou de loin, les habitants de la ville à l'exercice de planification, ne serait-ce que pour faire en sorte que les interventions répondent aux aspirations et aux besoins de ceux pour qui elles sont destinées.

\section{Retour sur quelques théories de la planification}

L'émergence de la planification contemporaine a été facilitée par l'établissement de politiques publiques et de marchés qui légitimaient l'action planificatrice et servaient de canaux de contrôle et de simplification de la vie collective. Contrôle, simplification et analyse étaient alors les grandes lignes directrices de la profession de planification, du moins dans le monde occidental. Certes, cette conception de l'ordre a varié à travers les époques et selon l'importance attribuée à chacune des sphères couvertes par l'art de la planification que sont les fondements rationnels et techniques de l'aménagement, le soutien au développement, la compréhension des relations sociales et les préoc- cupations esthétiques. Il reste que les planificateurs de toutes les époques ont travaillé en poursuivant une certaine idée de l'ordre social et urbain

Du début du XX ${ }^{\mathrm{e}}$ siècle jusqu'aux années 1940, l'ordre correspondait à l'organisation de la ville autour de principes de salubrité, de fonctionnalité et d'efficacité. Les planificateurs étaient des maîtres de la technique à la recherche de solutions rationnelles aux problèmes urbains et se gardant bien de prendre position dans les débats sociaux. Ils devaient s'engager à servir le bien civique en mettant de côté leurs émotions, leur subjectivité et leur appartenance à des groupes particuliers.

Des années 1940 aux années 1960, les planificateurs ont nourri cette même conception de la planification socialement neutre et rationnelle, tout en s'intéressant davantage aux enjeux physiques et territoriaux ${ }^{4}$. Les académiciens sortirent des universités et s'engagèrent davantage sur le terrain. C'est ainsi qu'on assista, à la fin des années 1940 et par la suite, à l'émergence de nouvelles préoccupations liées aux transformations de la ville. En Amérique du Nord et en Europe du moins, le mouvement de l'urban renewal était lancé afin de résoudre l'éventail des problèmes rencontrés par les aires urbaines physiquement dégradées et socialement défavorisées (slums and blighted areas) ${ }^{5}$. Des critiques ont rapidement souligné les effets pervers de tels programmes de renouveau urbain, notamment le remplacement d'un type d'habitat et d'une catégorie de population par des usages autres que résidentiels ou des catégories sociales plus aisées.

Au cours des années 1960, le constat de l'échec des grands projets modernistes de réhabilitation et de rénovation à améliorer les conditions de vie et de logement des populations défavorisées amena des intellectuels et des intervenants de la société civile à interroger les pratiques de planification et leur influence sur les morphologies urbaines. Conjuguée, en Amérique du Nord, à la critique radicale du phénomène d'étalement urbain, cette remise en question de la planification urbaine s'amplifia durant les années 1970 alors que la voix et le rôle des acteurs sociaux et des acteurs économiques dans le développement urbain gagnaient en importance sur la scène municipale. Les impacts de l'étalement urbain étaient discutés, entre autres les coûts de l'étalement et la production de formes urbaines socialement ségréguées. En même temps, des groupes sociaux, souvent représentants de minorités, réclamaient que les interventions issues de 
la planification urbaine tiennent davantage compte de leur réalité propre. Par exemple, aux États-Unis, Beauregard a souligné le fait que la planification des quartiers occupés par une population majoritairement noire soit assurée par des planificateurs blancs. Ainsi, les critiques relatives à l'absence de considérations des particularismes et des spécificités se firent plus nombreuses au cours des années 1970.

\section{Assurer la participation par-delà la communication}

Les revendications pour une prise en compte de la diversité sociale et des préoccupations des acteurs locaux dans la planification ouvraient la porte à une participation accrue de la population au processus planificateur durant les décennies suivantes. Les autorités publiques étaient alors de plus en plus convaincues que la participation de la population à la réflexion et au processus de planification urbaine ne pouvait que renforcer la légitimité sociale et politique des décisions. C'est pourquoi les expériences de planification se multiplièrent ainsi que les recherches menées pour mieux les comprendre et les évaluer.

Parmi les nouvelles approches à voir le jour ces dernières années, les approches associées à la critique postmoderne considèrent que le planificateur est un membre à part entière de la société civile et que son travail ne peut qu'être teinté par son appartenance à une ou à des communautés particulières ${ }^{6}$. Le rôle de ce planificateur serait alors d'ouvrir la perspective aux distinctions de genre, de race et d'orientation sexuelle laissées en marge dans la planification rationnelle. Une telle façon de faire consisterait, dans l'optique développée par Leonie Sandercock, à prendre en compte les histoires et les mémoires particulières (storytelling) des groupes marginalisés ${ }^{7}$. Cette approche de la planification développée à partir des années 1980 s'appuie sur les principes de justice et d'équité sociale.

Allant un peu dans le même sens, mais restant liée à l'idée d'une forme de rationalité guidant la planification, l'approche concertée ou communicationnelle (communicative ou collaborative planning) a mis l'accent sur la communication et les interactions réciproques entre les planificateurs et les acteurs sociaux ${ }^{8}$. Dans cette optique, les informations et les savoirs détenus par les citoyens sont tenus pour essentiels à la bonne réussite de la démarche. Dans des contextes institutionnels complexes et dynamiques, la planification urbaine communicationnelle serait influencée par des forces économiques, sociales et environnementales globales, ce qui favoriserait, selon ses promoteurs, une forme de gouvernance par laquelle les acteurs individuels et communautaires assument collectivement leur devenir. Ces derniers y trouveraient une façon de situer leurs intérêts dans les débats sociaux, voire de les positionner dans un cadre plus large.

Par contre, il est permis de se demander si de telles expériences n'accordent pas trop d'attention au processus comme tel, en focalisant sur la relation entre les planificateurs et les divers acteurs sociaux et ce, au détriment des questions pratiques que les processus de planification sont précisément chargés de traiter. En d'autres mots, les approches communicationnelles misent-elles plus sur les processus de planification (la forme) que sur les objets de l'aménagement, comme le logement, les aménités urbaines ou les services publics (le contenu) ? Une des critiques adressées à l'approche communicationnelle est qu'en misant sur la recherche d'un discours idéal inspiré de la théorie de l'agir communicationnel d'Habermas, les planificateurs négligeraient le fait que la négociation et l'interaction ne permet pas toujours d'apporter une solution à tous les problèmes ${ }^{9}$. En réponse à ces critiques, les tenants du courant communicationnel rappellent que l'objet premier des expériences concertées et d'élaboration de consensus (consensus building) est le dialogue authentique (authentic dialogue) ${ }^{10}$. Pour les tenants de ce courant, l'échange et la discussion ne sacrifient pas le contenu de la planification au profit de la procédure. Ils stipulent, par ailleurs, que l'élaboration des ententes a justement pour objet de trouver des solutions nouvelles, tout en favorisant le développement de compétences chez les acteurs et d'alliances entre eux. Un certain relativisme se dégage de cette position d'après laquelle les solutions diffèrent selon les personnes ou les groupes sociaux.

Ainsi, la place et le rôle du planificateur se sont modifiés au rythme des transformations qui ont traversé la société. L'acceptation de positions variées sur le rôle du planificateur se fait en parallèle de la reconnaissance de la diversité culturelle, des inégalités sociales et de la diversité des besoins. Le planificateur neutre et maître de la technique de la première moitié $d u X^{\mathrm{e}}$ siècle, d'ailleurs chargé d'élaborer des plans globaux ou des projets spécifiques commandés par les pou- 
voirs publics, a fait place à un interlocuteur à l'affût des différents systèmes de valeurs présents dans la société, s'inspirant des connaissances propres à chacun des acteurs et nourri de savoirs disciplinaires divers. En somme, la planification se présente aujourd'hui comme une activité à la confluence du savoir expert et des débats sociaux.

\section{La planification mise au défi de l'intégration}

Les approches participatives et communicationnelles ont largement inspiré les décideurs publics lors de l'élaboration de politiques et de programmes publics. Ceux-ci sont d'ailleurs produits et administrés dans la perspective de la formation de réseaux et de coalitions que Vincent Lemieux définit comme «des alliances temporaires et négociées d'acteurs individuels ou collectifs qui cherchent à obtenir des avantages plus grands que ceux qu'ils auraient obtenus hors de ces coalitions $\gg{ }^{11}$. Parallèlement, des individus et des collectivités tendent à agir de façon à modifier et améliorer leur cadre de vie et à interférer sur les conditions défavorables qui pèsent sur leur environnement. Ainsi, les acteurs publics et ceux de la société civile se rejoignent pour développer des partenariats qui, dans le cas des questions urbaines, peuvent prendre la forme de réseaux territorialisés à l'échelle des quartiers.

Par ailleurs, il est reconnu que ces approches concertées et intégrées, qui sont soutenues par les pouvoirs publics, font en sorte de déléguer vers le local une partie des pratiques de planification, du moins celles qui touchent le développement social du quartier ainsi que le cadre de vie. Des pans de la politique publique de lutte à la pauvreté se trouvent ainsi canalisés vers des instances locales. Il en va de même de l'accès aux ressources, tant matérielles que sociales, que possèdent les quartiers. Cependant, la délégation se limite bien souvent à l'administration locale de programmes existants ou à la mise en place de structures partenariales sans ajout de nouvelles ressources financières ni d'outils suffisamment puissants pour modifier des situations anciennes et structurelles.

\section{Les raisons de l’intégration}

Dans une étude récente sur le programme de revitalisation urbaine intégrée à Montréal, Divay et al. ${ }^{12}$ mentionnaient que le caractère intégré des politiques

renvoie simplement à une volonté d'aborder les problèmes en ne négligeant aucune de leurs dimensions et en tenant compte de l'ensemble des aspects de la vie des individus et des familles. Une telle mobilisation des différents secteurs d'activités et des réseaux sociaux s'inscrit dans une perspective néo-possibiliste - pour reprendre ce terme de la géographie - qui sousentend que l'action des individus et des collectivités peut améliorer l'environnement local et interférer sur les conditions défavorables qui pèsent sur la santé des collectivités et des personnes. Par contre, la participation de la société civile à l'intérieur de processus formels, notamment des politiques et des programmes publics, et dont l'une des fonctions est la régulation sociale, ne peut être tenue comme le gage d'une collaboration entière des acteurs sociaux, voire de tous les acteurs des milieux concernés. Ces processus participatifs qui sollicitent des acteurs de statuts divers, engagés dans des organismes de toutes sortes et intéressés par différents aspects de la vie sociale n'épuisent pas, loin s'en faut, les tensions et les conflits qui traversent la société. Ceux-ci sont projetés dans les processus de planification et de concertation qui sont tenus, dès lors, de les traiter d'une façon ou de l'autre.

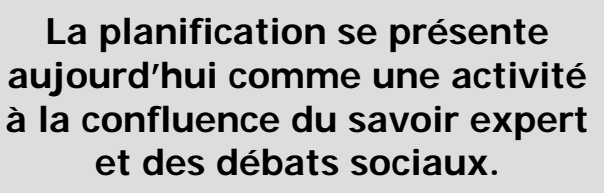

Les processus de planification urbaine ainsi que la formulation des politiques publiques se forment bien souvent en raison des problèmes sociaux et au contact des protestations et des actions engagées par des acteurs sociaux défendant autant des intérêts particuliers que des idéaux indépassables comme l'éradication des inégalités sociales ou l'atteinte du développement durable. Dans cette optique, les structures partenariales de planification ont pour principal objectif de négocier des normes et de proposer des objectifs qui seront récupérés dans l'offre de services gouvernementaux et de programmes publics. Elles ont aussi pour fonction de faire valoir des points de vue qui discréditent les lectures trop exclusivement économiques ${ }^{13}$ et de formuler des enjeux favorables aux groupes marginalisés. Elles tendent, enfin, à unifier la démarche en identifiant des priorités et en opérant des choix sur une base commune. 
De ce point de vue, les instances de participation publique forment des espaces de dialogue et de démocratie civile dont la fonction est d'agréger des intentionnalités exprimées lors des différentes étapes du processus de planification et de les traduire en un projet partagé. En se plaçant en situation de négociation et de transaction, les acteurs sociaux confrontent les agents publics de la planification et projettent leurs revendications ou leurs projets dans un espace public plus large dans lequel les autres grandes composantes du système d'acteurs se trouvent mobilisés. Ces espaces de concertation, chargés de la planification de quartier, peuvent aussi apparaitre comme des espaces transactionnels au sens où l'entendent Jean Rémy et Maurice Blanc dans la mesure où ils sont chargés de négocier des situations de conflit paraissant insolubles ${ }^{14}$. Formulée de la sorte, l'analyse transactionnelle permet d'aborder les instances de concertation locale en planification urbaine comme des formes sociales de l'échange et des modes de structuration des rapports de force observables dans toutes tentatives de régulation de conflits ou de tensions sociales. De tels échanges sont abordés, selon cette approche, non plus sous un mode dual ou bipolaire, par exemple entre l'État et la société civile ou entre les patrons et les travailleurs à l'intérieur d'une organisation, mais dans la perspective des réseaux et des coalitions. Il importe alors de traduire, à l'intérieur d'un espace public de concertation, la complexité grandissante des systèmes d'acteurs et des rationalités multiples qui sous-tendent l'action collective. La transaction est ainsi constituée de différentes phases, soit le conflit, la coopération, la négociation et la régulation. La coopération se fait dans un contexte normé et régulé par la recherche d'un intérêt commun dont les termes sont sans cesse discutés et négociés, mais déterminés par des intérêts en compétition et en conflit. On soutient ainsi que l'analyse des transactions sociales permet de comprendre les compromis établis dans le réseau d'acteurs sociaux et les formes de la négociation qui ont conduit à tel consensus supposé. En d'autres mots, le processus de planification intégrée et concertée consiste à transiger autour des conflits et des tensions présents dans le cadre du quartier.

\section{Intégration et transaction sociale : le cas de la RUI dans le quartier Sainte-Marie à Montréal}

À Montréal, les acteurs locaux ont développé, et de longue date, des aptitudes à formuler des projets et des stratégies de revitalisation urbaine. Les nombreux portraits de quartier et plans d'action qui s'ensuivirent ont essaimé des projets de logements sociaux ou de centres communautaires sur le territoire montréalais, pour ne nommer que ces exemples-là. Les réseaux communautaires de quartier ont ainsi fait la preuve d'une expertise et d'un savoir hors du commun. Ces plans ont été produits dans le but d'orienter le développement à des fins d'améliorer les conditions sociales et environnementales du quartier. Des groupes locaux ont ainsi engagé des démarches d'empowerment et de concertation à l'échelle des quartiers. Ils ont cherché à atténuer, sinon corriger les effets de milieu (inégalités sociales, stress locaux, incertitudes environnementales) qui pèsent sur la qualité de vie et la santé des résidents. Dans bien des cas, ils sont parvenus à mettre en œuvre une partie de leur plan d'action, parfois de façon spectaculaire, et à obtenir des résultats notables. En d'autres cas, les résultats se font encore attendre.

Le programme de revitalisation urbaine intégrée (RUI) de la Ville de Montréal est venu en quelque sorte s'ajouter à des démarches communautaires déjà riches en ce domaine. Il a justement été conçu dans l'optique des approches de planification concertée (ou participative) ouvertes aux problématiques multidimensionnelles et couplées aux réseaux sociaux existants. Et il a fait appel aux groupes locaux déjà occupés dans des expériences de revitalisation urbaine, tout en cherchant à élargir la base des acteurs interpellés.

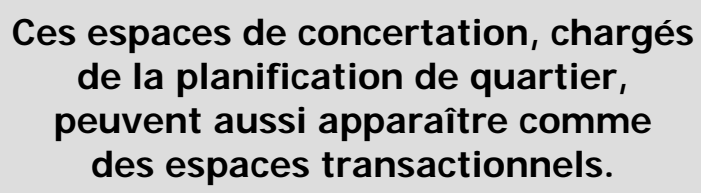

Les structures de concertation développées à l'intérieur du programme de la RUI ont fait en sorte d'endiguer les démarches communautaires de revitalisation existantes à l'intérieur de processus formalisés de concertation et de coopération.

Il faut indiquer que le mandat des comités de revitalisation locale, formés pour mener à terme la démarche de revitalisation, était d'établir un portrait de quartier, de déterminer les enjeux du développement et d'ébaucher un plan d'action allant dans le sens de la revitalisation et la lutte aux inégalités sociales. 
L'objectif implicite de la démarche était, généralement, d'élaborer des solutions socialement acceptables pour le plus grand nombre et d'arriver ainsi à des compromis négociés.

Sur le fond, la Ville se donna une définition de la revitalisation urbaine très sociale. La RUI est définie comme un moyen d'améliorer « les conditions socioéconomiques et le cadre de vie d'un territoire défavorisé donné », en partant d'une vision globale et à long terme d'une action concertée, donnant une large part à la population, agissant sur tous les facteurs de pauvreté et ce, en intégrant des actions spécifiques ${ }^{15}$. Le champ d'intervention de la RUI couvre ainsi un vaste éventail de problématiques, notamment la lutte aux inégalités sociales, l'abordabilité du logement, l'amélioration du cadre de vie et de la structure d'opportunités (la sécurité dans les lieux publics, l'accès aux équipements, services et lieux d'activité ou de consommation), la sécurité alimentaire, l'amélioration de la littératie, etc.

Que pouvons-nous conclure à la lumière de la première phase de la RUI dans le quartier Sainte-Marie, celle précisément chargée de l'élaboration du plan d'action? D'abord, il faut reconnaître que le planificateur urbain a revêtu certains des habits du postmodernisme sans toutefois recourir à tous les aspects de ce courant : les solutions recherchées devaient tenir compte de la diversité des points de vue tout en reflétant les débats sociaux et culturels qui ont émaillé les discours des différents acteurs. Cela dit, le processus fut confiné à un comité formé en gros des différents collèges qui se partagent habituellement les espaces de concertation montréalais. Les places étaient déterminées en fonction des structures existantes. Il n'en reste pas moins que la population a été sollicitée lors d'assemblées publiques, mais sans que les voix marginales ne trouvent nécessairement à s'exprimer de façon particulière. Cette structure de concertation formalisée à proximité des instances de la Ville a pris le relais de démarches de revitalisation menées par des groupes communautaires, mais sans réussir complètement à associer les secteurs de la santé et de l'éducation. L'élargissement du réseau de partenaires apparaît encore inachevé.

Sur un autre plan, les grands enjeux ont été traités sans que soit dégagée la nature conflictuelle des problématiques : comment concilier le développement résidentiel privé et les besoins de logements sociaux et abordables ? Comment développer les espaces vacants de nouveaux logements, accroître la population $\mathrm{du}$ quartier et assurer le maintien des résidents actuels ? Comment donc éviter les effets pervers du développement, dont la gentrification? Comment maintenir les services aux personnes en grande difficulté comme les consommateurs de drogues fortes, les prostitués et les itinérants, tout en accédant aux demandes de résidents en faveur de l'intensification des mesures de sécurité dans les espaces publics ? Comment concilier, enfin, les besoins des différents segments de la population du quartier? Restées sans réponse, ces questions n'ont toutefois pas été totalement oblitérées. Formulées comme des enjeux, elles signifient fortement les tensions profondes qui traversent l'évolution récente des quartiers anciens. Le plan d'action est d'ailleurs une sommation des enjeux sans que des priorités ne soient établies. Elles le seront à des étapes subséquentes mais, durant la première année de l'expérience, le pari était de ne pas aborder les situations de conflit lors des rencontres du comité de revitalisation locale. En d'autres termes, l'espace transactionnel est resté extérieur au processus de la RUI.

\section{Conclusion}

Les expériences montréalaises de RUI sont encore embryonnaires. Elles ont fait appel aux concepts de participation et d'intégration dans la perspective croisée de la lutte aux inégalités sociales et de la revitalisation urbaine des quartiers anciens. Pour l'essentiel, elles démontrent que le recours des approches participatives et communicationnelles en planification a gagné l'administration municipale. Le planificateur municipal fait dorénavant partie d'un processus partenarial qui ne lui reconnaît pas de statut privilégié mais lui confère, en revanche, une légitimité populaire insoupçonnée. Cette nouvelle légitimité sera toutefois mise à rude épreuve devant l'imminence des choix stratégiques qu'il faudra bien établir et le réveil des tensions que cette opération ne manquera pas d'occasionner.

Le programme de la RUI a porté les deux drapeaux des nouvelles théories de la planification: participation et intégration. Sur le plan de la participation, il faut reconnaître que la participation confinée, telle que pratiquée dans Sainte-Marie, a permis jusqu'ici de reporter à plus tard le traitement des situations épineuses. Cela ne pourra durer. Par ailleurs, le processus 
public n'a pas tout à fait permis la formation d'un espace public élargi, observable dans les médias par exemple, faisant en sorte que les enjeux de la RUI n'ont pas connu un large écho à l'échelle du quartier. Sans cet espace public ouvert, il sera difficile de prétendre à un plan d'action décidé et partagé par tous les segments de la population du quartier. Enfin, il faut noter que la voix des populations marginales s'est fait peu entendre jusqu'ici.

Sur le plan de l'intégration, rappelons que des secteurs clés comme l'éducation et la santé sont encore en marge. D'ailleurs, les stratégies établies dans le quartier sont encore formulées de manière sectorielle. La RUI prétendait agir de manière intégrée en formulant par exemple que l'amélioration des conditions de logement ou d'employabilité devaient se répercuter de manière bénéfique sur les services sociaux et le système scolaire qui, en revanche, devaient contribuer à l'amélioration de l'état de la population du quartier. Les actions en santé, en éducation ou en aménagement devaient donc être pensées de manière commune. Cette intersectorialité triomphante est toujours formulée comme un objectif, mais son articulation pratique reste à établir.

Le bilan de la première étape du processus a été globalement positif. L'apprentissage n'est toutefois pas achevé. Et l'un des enjeux est précisément la maîtrise de ces nouvelles approches en planification.

\section{Remerciements}

Les auteurs soulignent la contribution de Patrick Herjean à l'étude sur l'expérience de la RUI dans le quartier Sainte-Marie.

\section{Notes et références}

1 Gilles Sénécal est professeur à l'INRS-Urbanisation, Culture et Société. Ses travaux de recherche portent sur les questions d'environnement urbain et de la qualité de vie en ville. Il s'intéresse particulièrement aux expériences de revitalisation urbaine. Geneviève Cloutier est étudiante au doctorat en Études urbaines à l'INRS. Elle travaille sur les interactions entre les acteurs participant aux processus de planification de la revitalisation urbaine des quartiers centraux.

2 Ville de Montréal (2003). L'approche de revitalisation urbaine intégrée et les facteurs de réussite, document Power Point.
3 Beauregard, R.A. (1998). « Writing the Planner», Journal of Planning Education and Research, vol. 18, p. 93-101; Beauregard, R.A. (2001). "The Multiplicities of Planning », Journal of Planning Education and Research, vol. 20, p. 437-439. Innes, J.E. (1998). «Information in Communicative Planning », Journal of the American Planning Association, vol. 64, $\mathrm{n}^{\circ}$ 1, p. 52-63. Fishman, R. (1980). "The Anti-planners: The Contemporary Revolt against Planning and its Significance for Planning History », dans G.E. Cherry (dir.), Shaping an Urban World, Londres, Mansell, p. 243-252; Zukin, S. (1998). « Urban Lifestyles: Diversity and Standardization in Spaces of Consumption », Urban Studies, vol. 35, $\mathrm{n}^{\circ}$ 5-6, p. 825-839.

4 Birch, E.L. (2001). «Practitioners and the Art of Planning ", Journal of Planning Education and Research, vol. 20, p. 407-422.

5 Greer, S. (1965). Urban Renewal and American Cities, The Bobbs-Merrill Company; Teaford, J.C. (1990). The Rough Road to Renaissance, Baltimore, The Johns Hopkins University Press.

6 Hamel, P. (1997). « La critique postmoderne et le courant communicationnel au sein des théories de la planification: une rencontre difficile », Cahiers de géographie du Québec, vol. 41, n 114, p. 311-321.

7 Beauregard, R.A. (1998), op. cit.

8 Healey, P. et J. Coaffe (2003). « My Voice: My Place: Tracking Transformations in urban governance », Urban Studies, vol. 40, n 10, p. 1979-199; Innes (1998), op. cit.

9 Fainstein, S.S. (2000). « New Directions in Planning Theory », Urban Affairs Review, vol. 35, n 4, p. 451-478.

10 Innes, J.A. et D. Booher (2004). « Reframing Public Participation: Strategies for the $21^{\text {st }}$ Century », Planning Theory \& Pratices, vol. $5, n^{\circ} 4$, p. 419-436.

11 Lemieux, V. (2002). L'étude des politiques publiques, les acteurs et leur pouvoir, Québec, Presses de l'Université Laval, $2^{\mathrm{e}}$ édition, p. 55.

12 Divay, G., P.J. Hamel, D. Rose, A.-M. Séguin, G. Sénécal et P. Bernard (2004). Projets pilotes de revitalisation urbaine intégrée: démarche d'évaluation, INRS-Urbanisation, Culture et Société, 213 p. Le programme de revitalisation urbaine intégrée de la Ville de Montréal consista à lancer trois expériences pilotes dans les quartiers de Sainte-Marie, Saint-Pierre et dans le secteur Galt. Depuis, les expériences de Montréal-Nord et de Saint-Michel se sont ajoutées.

13 Lemieux, V. (2002). op. cit, p. 79.

14 Blanc, M. et al. (1995). Pour une sociologie de la transaction sociale, Paris, L'Harmattan.

15 Ville de Montréal, dans Divay et al. (2004). op. cit. 


\section{Publicité}

« Maîtrise en gestion de projet » 